\title{
Vectorial analysis of the electrocardiogram from conventional limb leads in healthy adult Nepalese females
}

\author{
Rajesh Prajapati ${ }^{* 1}$, Neebha Amatya $^{1}$, Rajab Rana Magar ${ }^{1}$, Ripti Shrestha ${ }^{1}$ \\ 'Department of Physiology, Gandaki Medical College Teaching Hospital
}

\begin{abstract}
Introduction: Electrocardiogram interpretation plays a vital role in the initial evaluation of patients presenting with cardiac complaints. Assessment of degree of cardiac vector is one of the important parameters to be studied in electrocardiogram. Methods: A prospective cross-sectional study was carried out among 84 female subjects, aged 18 to 40 years of age, including students and staffs of Gandaki Medical College Teaching hospital and Research Center, Pokhara, Nepal over a period of one month from 1st October to 1st November 2021. A Standard electrocardiogram machine was used and the electrocardiogram was recorded using the conventional limb leads. The individual mean electrical axis of the heart was plotted using the net voltage of QRS complex of Lead-I and Lead-III. The possible correlation between cardiac vector and physical measurements like height weight, body surface area and body mass index were analyzed. The data were analyzed in Statistical Package for the Social Sciences version 27.0. Results: The normal mean electrical axis of the healthy female subjects was observed as $61.7 \pm 23.51^{\circ}$. There was a significant positive correlation of cardiac vector with height $(\mathrm{p}<0.05)$, whereas a negative correlation was observed with weight and body mass index ( $<<0.01)$. However, there was no significant correlation with body surface area. In our study, we observed the maximum left axis cardiac vector as $-2^{\circ}$ and right axis as $98^{\circ}$ among 84 female subjects. Conclusions: Documentation of cardiac vector was made using standard bipolar limb leads in normal healthy female subjects. Body mass index is involved in the deviation of cardiac vector with a negative correlation. This observation could make it quite attractive for use in clinical practice.
\end{abstract}

Keywords: Axis deviation, body mass index, cardiac vector, electrocardiograph.

\section{*Correspondence:}

Dr. Rajesh Prajapati, PhD

Lecturer, Department of Physiology,

Gandaki Medical College Teaching Hospital,

Pokhara, Nepal

Email: razpra7@gmail.com

Submitted: November 3, 2021

Accepted: December 8, 2021

To cite: Prajapati R, Amatya N, Magar $\mathrm{RR}$, Shrestha R. Vectorial analysis of the electrocardiogram from conventional limb leads in healthy adult Nepalese females. JGMC Nepal. 2021;14(2):84-7.

DOI: 10.3126/jgmcn.v14i2.40656

\section{INTRODUCTION}

Electrocardiography is used to elucidate cardiac arrhythmias and conduction defects, and to diagnose and localize myocardial hypertrophy, ischemia and infarction. ${ }^{1}$ Electrocardiogram (ECG) interpretation plays a vital role in the initial evaluation of patients presenting with cardiac complaints. ${ }^{2}$ The concept of cardiac vector describing the electrical activity of the heart and the three cardinal bipolar limb lead vectors (lead I, lead II \& lead III) forming an equilateral triangle with heart at the centre of the homogeneous volume spherical conductor was first described by Einthoven, the father of Electrocardiography even before a century. ${ }^{3}$ Assessment of degree of axis of mean QRS vector (mean electrical axis of the heart) is one of the important parameter to be studied in ECG, as it provides information about the conduction defects and also the hypertrophy of the ventricles of heart. ${ }^{4}$

A lot of advancements had come in the ECG machine, yet the basic physics principle of ECG is not clearly understood. The attempt by various other researchers in trying to solve the relationship between 
Heart and Lead vector was incomplete. The normal QRS (Vector) axis is $-30^{\circ}$ to $90^{\circ 5}$ and many published reference values are available like the one from India where they have reported the electrical axis of the heart at an angle of $68.93^{\circ}$ in their population. ${ }^{6}$ However, the inappropriate use of control data obtained from non-native populations is a major cause of misinterpreting ECG results. Further, vector analysis of the ECG remains a major biological variable that varies from individual's characteristics to environmental parameters as well as wide variations in socio-economic, geographical, climatic and nutritional conditions. ${ }^{7}$ There are several potential sources of variability in a parameter ranging from individual characteristics (e.g. gender, age, body size, race, level of regular physical activity, circadian rhythms) to environmental (e.g. socio-economic states, exposures to altitude, smoking history, posture and technical aspects). ${ }^{8}$

In an attempt to reduce the variability and improve accuracy, the use of reference values from a geographically related population has been strongly recommended and to the best of our knowledge, no such reference values have been set for cardiac function test in this country. There exist wide gaps in our knowledge of "norms" for Nepalese for different cardiac function tests used in clinical medicine. A great need, therefore, exists to collect more data on normal in order to bridge up these gaps in our knowledge. The axis of cardiac vector results are reference values obtained from quantified studies of normal values in healthy population. ${ }^{9}$ Therefore, it is necessary to establish our own mean electrical axis of the heart for the assessment of cardiac function test. Hence, in this study we have established the electrical axis of the heart of the healthy adult Nepalese females.

\section{METHODS}

This is a prospective cross sectional study carried out among 84 female subjects, aged 18- 40 years of age, including students and staffs of Gandaki Medical College Teaching hospital and Research Center, Pokhara, Nepal over a period of one month from $1^{\text {st }}$ October to $1^{\text {st }}$ November 2021.Ethical clearance was obtained from the Institutional ethical review committee board, Gandaki Medical College (Ref no::22/78/79). All the recordings were taken in the skill lab in the Department of Physiology under laboratory condition $\left(26 \pm 2^{\circ} \mathrm{C}\right)$. The subjects were given a detailed explanation of the study and were asked to sign a written consent form. A written consent includes the detail information about the name, height, weight, age, Body Surface Area (BSA) and Body Mass Index (BMI).The relevant alcoholic history and smoking habits were elicited from each subject to make sure that the subjects included in the study satisfied the criteria of normal and healthy individual. The subjects were apparently fit, receiving no medication at the time of the study. Standard ECG machine (MAC 600, GE MSIT, Inc., USA) was used and the ECG was recorded using the conventional limb leads. The individual mean electrical axis of the heart was plotted using the net voltage of QRS complex of Lead-I and Lead-III. ${ }^{10}$ ECG reading was carried out by single observer in order to eliminate interpreter bias. BSA was recorded in sq. $\mathrm{m}$. from the subject's height and weight by reference to the nomogram prepared by Boothby and Sandiford, based on Dubois's formula, ${ }^{11}$

BSA = Weight $(\mathrm{kg})^{0.425} \times$ Height $(\mathrm{m})^{0.725} \times 71.84$

BMI was calculated from the standard formula i.e. $\mathrm{BMI}=$ weight $(\mathrm{kg}) /$ height $(\mathrm{m})^{2}$.

Different dependent and independent parameters of the normal female subjects were recorded and analyzed for the purpose of establishing a normative database of mean electrical axis. The possible correlation between axis of cardiac vector and physical measurements like height, weight, BSA and BMI, which could finally help in proposing the reference for analyzing vector in ECG, was also analyzed.

The $\mathrm{P}$ value less than 0.05 was considered statistically significant. All analysis were performed with the Statistical Package for Social Sciences version 27.0.

\section{RESULTS}

During the study period from $1^{\text {st }}$ October to $1^{\text {st }}$ November 2021, 84 Nepalese female subjects, aged 18 to 40 years of age were enrolled in this study. Initially their cardiac vector was observed followed by the analysis of correlation between the cardiac vector and physical parameters.

\section{Mean electrical axis (cardiac vector):}

The normal mean electrical axis of the healthy female subjects was observed as $61.7 \pm 23.51^{\circ}$, which is shown in the figure 1.

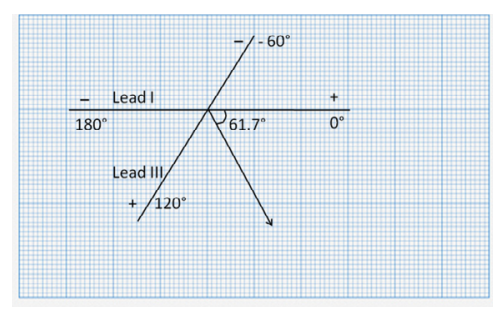

Figure 1: Plotting the mean electrical axis of the heart from lead I and III of ECG from conventional limb leads 


\section{Correlation between cardiac vector and physical} parameters:

Correlation analysis of cardiac vector with different physical parameters showed different relationship (Table 1). There was significant positive correlation with height $(\mathrm{P}<0.05)$, whereas negative correlation was observed with weight and $\mathrm{BMI}(\mathrm{P}<0.01)$.

Table 1: Correlation coefficients between cardiac vector and physical anthropometric variables

\begin{tabular}{ccccc}
\hline Parameters & Height & Weight & BSA & BMI \\
Cardiac vector & $0.227^{*}$ & $-0.383^{* *}$ & -0.160 & $-0.503^{* *}$ \\
\hline
\end{tabular}

*Correlation is significant at the 0.05 level

${ }^{* *}$ Correlation is significant at the 0.01 level

3. Effect of BMI on the mean electrical axis:

We observed that BMI showed negative correlation with cardiac vector $(p<0.01)$ hence we categorized the BMI under nutritional status based on World health organization and observed the cardiac vector. The table 2 showed the cardiac vector towards the right axis with low BMI whereas left axis with high BMI. The distribution of cardiac vector with the change in the BMI is shown in figure 2. As the BMI increases the cardiac vector decreases showing the negative correlation.

Table 2: The relation of BMI with the cardiac vector

\begin{tabular}{ccc}
\hline BMI & Nutritional status based on WHO & Vector angle Mean \pm SD \\
14 to 18.4 & Underweight & $83.16 \pm 7.98^{\circ}$ \\
18.5 to 24.9 & Normal & $62.5 \pm 20.46^{\circ}$ \\
25 to 31 & Overweight & $36.34 \pm 25.19^{\circ}$ \\
\hline
\end{tabular}

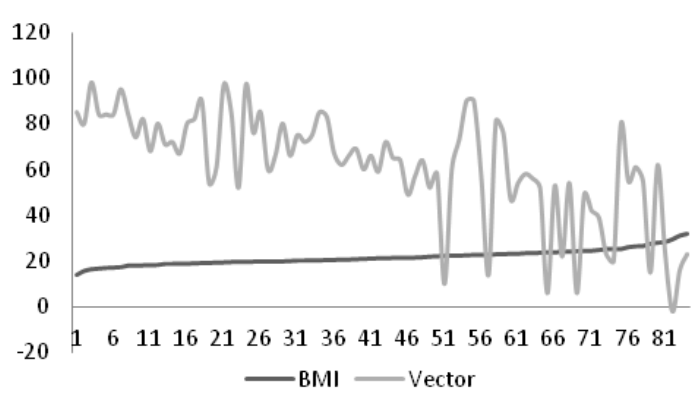

Figure 2: Distribution of cardiac vector with the change in the BMI

4. The right axis and left axis cardiac vector and BMI:

In our study, we observed the maximum left axis cardiac vector as $-2^{\circ}$ and right axis as $98^{\circ}$ among 84 female subjects. We then analyzed their BMI and found it to be 29.57 and 16.66 respectively. This analysis strengthens the negative correlation of cardiac vector with BMI.

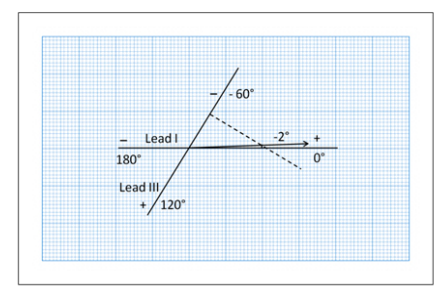

A.

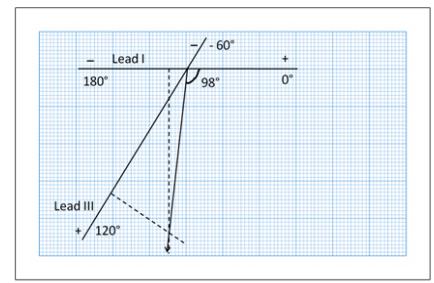

C.

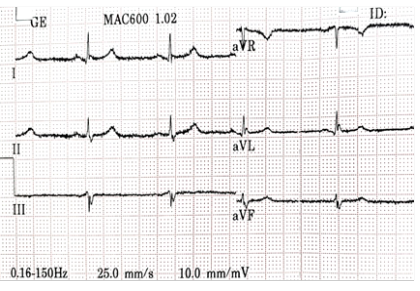

B

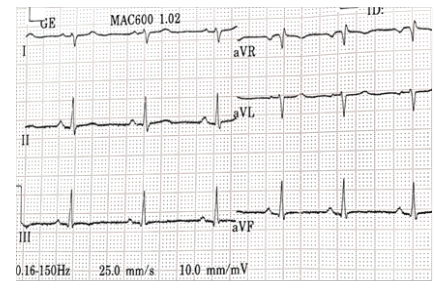

D.
Figure 3: Representative figure showing left and right axis of cardiac vector with representative ECG graph. (A) The mean electrical axis of the heart from lead I and III of ECG from conventional limb leads showing left axis $\left(-2^{\circ}\right)$. (B) The representative ECG of figure A. (C)The mean electrical axis of the heart from lead I and III of ECG from conventional limb lead showing right axis $\left(98^{\circ}\right)$. (D) The representative ECG of figure $\mathrm{C}$.

\section{DISCUSSION}

ECG is one of the most important diagnostic tool for appropriate interpretation and diagnosis of cardiac disease. The understanding of the Einthoven's triangle hypotheses is central to the field of electrocardiography, but the concept of cardiac vectors is very difficult to understand. ${ }^{12}$ We observed that the axis of mean cardiac vector in our study is less than that observed in the Indian study. ${ }^{6}$ It may be due to differences among the populations, gender or technical and procedural issues. Hence, this research is a fundamental principle of the tools and programs to contribute to the development of health science.

For over a century, the cardiac field resists our detailed understanding, specifically in the areas of axis of cardiac vector and its relation with different physical and anthropometric parameters. ${ }^{13}$ In this study, there was a pronounced tendency toward the horizontal cardiac vector with increasing body weight and in general, the influence of relative body weight was more prominent to influence the cardiac vector. Hence, we conclude that BMI is novel key player for a more precise definition of normal electrocardiographic standards. It is clear from these data that, in healthy females, differences in relative fatness or leanness are reflected in characteristic differences in 
the electrocardiograms and affecting the axis of cardiac vector. However, the relative masses of the right and left ventricles, as well as the geometric position of the whole heart, are involved in the electrical axis. ${ }^{14}$ Sokolow et al. ${ }^{15}$ concluded that the shift to the left of the electrical position of the heart in obesity cannot be explained by anatomic position alone. Hence, from our study we can presume that the increase in BMI (overweight) causes the increase in left ventricle mass which leads to cardiac vector towards the left or horizontal position.

\section{CONCLUSIONS}

The documentation of cardiac vector was made using standard bipolar limb leads from conventional limb leads in normal healthy female subjects. The negative correlation characteristics of the cardiac vector with BMI are described. This observation greatly simplifies interpretation and provides a rationale and objective basis for the analysis of the electrocardiogram in clinical practice.

\section{ACKNOWLEDGEMENT}

We are grateful to Mr. Sandip Pahari, lecturer, Department of Community Medicine, Gandaki Medical College, for statistical analysis.

\section{CONFLICT OF INTEREST: None declared}

\section{SOURCE OF FUNDING: None}

\section{REFERENCES}

1. Singh PN, Sajjad MA. Simplified calculation of mean QRS vector (mean electrical axis of heart) of electrocardiogram. Indian J Physiol Pharmacol. 2003;47(2):212-6.

2. Majd A, Joseph L. A brief review: history to understand fundamentals of electrocardiography. J Community Hosp Intern Med Perspect. 2012;2(1):1-5. DOI: 10.3402/ jchimp.v2i1.14383 PMID: 23882360.

3. Malmivuo J, Plonsey R. Bioelectromagnetism - Principles and Applications of Bioelectric and Biomagnetis Fields. Chapter: 15. Oxford University Press. 1995;277-89. DOI:10.1093/acprof:oso/9780195058239.001.0001 PMID: 7494216.

4. Benjamin EJ, Wulff H, Widdicombe JH, Zheng J, Donald
MB, Jose LP. A simple device to illustrate the Einthoven triangle. Adv Physiol Educ. 2012;36(4):319-24. DOI: 10.1152/advan.00029.2012 PMID: 23209014.

5. Shamroth L. An introduction to electrocardiography. $7^{\text {th }}$ edition. Blackwell science ltd. 2010;7-172.

6. Rajini T, Samuel MD. Formulation and clinical application of cardiac vector hypotheses in ECG interpretation using vector physics principle. Ejpmr. 2018;5(11):523-36.

7. Yang Y, Zhang E, Zhang J, Chen S, Yu G, Liu X, et al. Relationship between occupational noise exposure and the risk factors of cardiovascular disease in China: A meta-analysis. Medicine.2018;97(30).DOI:10.1097/ MD.0000000000011720 PMID: 30045338.

8. Cheng CF, Kuo HC, Chien KR . Genetic modifiers of cardiac arrhythmias. Trends Mol Med. 2003;9(2):5966. DOI: $10.1016 / s 1471-4914(03) 00004-2$

9. Abdelraheem M, Selim H, Abdelhamid TK. Human identification using the main loop of the vector cardiogram. Am J Signal Process. 2012;2:23-9. DOI: 10.5923/J.AJSP.20120202.04

10. Fisch C, Braunworld E. In Heart Diseases. Electrocardiography. 1997;108-45.

11. Jepegnanam V, Amirtharaj G, Damodarasamy S, Rao VM. Peak expiratory flow rate in a random healthy population of Coimbatore. Indian J Physiol Pharmacol. 1996;40(2):127-33 PMID: 9062806.

12. Kittnar O, Mlček M. Analysis of the electrical heart field. Physiol Res. 2010;59(Suppl. 1):S19-S24. DOI: 10.33549/physiolres.932004 PMID: 20626216.

13. Batchvarov V, Malik M. Measurement and interpretation of QT dispersion. Prog Cardiovasc Dis. 2000;42:325-44. DOI: 10.1053/pcad.2000.0420325 PMID:10768311.

14. Ernst S, Ancel K. The effect of age and body weight on the electrocardiogram of healthy men. Circulation. 1952;5:749-61. PMID:12988354.

15. Sokolow M, Friedlander RD. The normal unipolar precordial and limb electrocardiogram. Am. Heart J. 1949;38:665-8. DOI: 10.1016/0002-8703(49)90525-6. 